\title{
A numerical study on the non-smooth solutions of the nonlinear weakly singular fractional integro-differential equations
}

\author{
Sayed Arsalan Sajjadi ${ }^{1}$, Hashem Saberi Najafi ${ }^{2}$, and Hossein Aminikhah ${ }^{3}$ \\ ${ }^{1}$ University of Guilan \\ ${ }^{2}$ Faculty of Mathematical Sciences, University of Guilan,P.O.Box 1914,Rasht,Iran \\ ${ }^{3}$ Univ Guilan
}

December 15, 2021

\begin{abstract}
The solutions of weakly singular fractional integro-differential equations involving the Caputo derivative have singularity at the lower bound of the domain of integration. In this paper, we design an algorithm to prevail on this non-smooth behaviour of solutions of the nonlinear fractional integro-differential equations with a weakly singular kernel. The convergence of the proposed method is investigated. The proposed scheme is employed to solve four numerical examples in order to test its efficiency and accuracy.
\end{abstract}

\section{Hosted file}

s.a.sajjadi.pdf available at https://authorea.com/users/451350/articles/549538-a-numericalstudy-on-the-non-smooth-solutions-of-the-nonlinear-weakly-singular-fractional-integrodi $\% E F \% A C \%$ erential-equations 